\title{
VULNERABILIDAD, RESILIENCIA Y ORDENAMIENTO TERRITORIAL DE LOS DESASTRES SOCIONATURALES EN CHILE ${ }^{1}$
}

\author{
Hugo ROMERO \\ Profesor del Departamento de Geografía \\ Director del Núcleo Milenio de Investigaciones sobre Vulnerabilidad Social de \\ los Desastres Socionaturales CIVDES). Universidad de Chile
}

Recibido: $17 / 04 / 2014$

"Pudieron ser muchos más los muertos si la gente no hubiera obedecido a sus instintos, en vez de esperar a los nervios de zombie del SHOA (Servicio Hidrográfico y Oceanográfico del Estado), el silencio oscuro de la Armada (rama especializada de las fuerzas armadas en asuntos marítimos), a la agitación de gallina sin cabeza de la Onemi (la Oficina Nacional de Emergencia dependiente del Ministerio del Interior) y al colapso de un Estado que en tres minutos ya no sabía quién era". ASCANIO CAVALLO, "La Noche del Laberinto”, $L a$ Tercera, 9 de febrero de 2013, 6-8.

Aceptado: 30/09/2014

RESUMEN: Las catástrofes naturales constituyen fenómenos de enorme trascendencia económica y espacial, lo que justifica la atención que merecen desde el punto de vista geográfico. De ahí el interés en conocer y valorar el significado que los desastres recientes han tenido en la transformación del territorio chileno, poniendo de manifiesto la situación de vulnerabilidad a la que se enfrentan el país y sus sociedades y, sobre todo, los altos costes que derivan de la ausencia de un ordenamiento territorial que, apoyado en el conocimiento y en la estimación rigurosos de las

1 Investigación desarrollada en el marco del Proyecto del Fondo de Desarrollo Científico y Tecnológico (Fondecyt) Nº1120204 y Milenio NS100022 del Ministerio de Economía, Fomento y Turismo de Chile. 
modalidades y de los niveles de riesgo existentes, neutralice o mitigue la gravedad de sus impactos. La variedad de manifestaciones y ejemplos lo demuestra con claridad a través de un muestrario de experiencias reveladoras de la magnitud del problema y de su persistencia en el tiempo. Y es que las insuficiencias y omisiones que en este sentido presenta la gestión pública del territorio - asociadas a una ideología escasamente sensible con las necesidades que le afectan - ofrece un fiel testimonio en la deficiente capacidad para afrontar con la necesaria garantía las múltiples y fuertemente lesivas dimensiones socionaturales y espaciales de la catástrofe.

PALABRAS CLAVES: Riesgo natural, ordenamiento territorial, política publica, impactos socionaturales

\section{VULNERABILITY, RESILIENCE AND SPATIAL PLANNING OF THE SOCIONATU- RAL DISASTERS IN CHILE}

ABSTRACT: Natural disasters are phenomena of enormous economic and spatial importance, justifying the attention they deserve from the geographical point of view. Hence the interest in knowing the meaning and value that recent disasters have had on the transformation of the Chilean territory, where it shows the vulnerability to the country and their societies face and especially the high costs arising from the absence of a territorial system that relied on the knowledge and the rigorous estimation methods and the levels of risk, neutralize or mitigate the severity of its impact. The variety of examples clearly demonstrated through a sample of revelatory experiences of the magnitude of the problem and its persistence over time. And is that the shortcomings and omissions in this sense presents public land management - a sparsely associated ideology sensitive to the needs that affect it - provides a faithful witness in poor ability to cope with the necessary assurance and heavily damaging multiple socionatural and spatial dimensions of the catastrophe.

KEY WORDS: Natural risk, Spatial Planning, Public Policy, Socio-naturals impacts

\section{I. INTRODUCCIÓN}

Chile, al igual que otros países latinoamericanos, ha sido severamente afectado por desastres "naturales" durante los años recientes. En los momentos de escribir este texto, el martes 1 de abril de 2014, un terremoto magnitud 8,2 en la Escala de Richter ha azotado el norte del país y obligado a evacuar a cerca de un millón de personas, desde sus hogares a los cerros cercanos, para ponerles a salvo de un probable tsunami, repitiéndose una situación similar al día siguiente. Asimismo destacan por su magnitud, amplitud y daños causados, el terremoto y el tsunami ocurridos el 27 de febrero de 2010, cuyo costo en vidas humanas superó las 500 víctimas, mientras que económicamente las pérdidas estimadas fueron mayores que treinta mil millones de dólares (cerca de un noveno del Producto Interno Bruto del país) (Romero et al., 2010 y 2011b). Otro desastre de gran impacto social y mediático había sido la erupción del Volcán Chaitén (localizado a $1.300 \mathrm{Km}$. al sur de Santiago), que tuvo lugar a partir del 1 de mayo de 2008, obligando a evacuar la totalidad de la población que residía en la ciudad 
del mismo nombre (alrededor de siete mil personas), muchas de las cuáles aún permanecen distribuidas en pueblos y ciudades de la región o bien han optado por retornar a sus antiguos hogares en la medida que ellos mismos han dado por superada la amenaza (Marchant, 2010; SAlgado y Ugarte, 2012). Además, una severa y persistente sequía, asociada al predominio del Fenómeno La Niña de los años 2011, 2012 y 2013, ha reducido la disponibilidad de nieve y agua para riego y producción de hidroelectricidad en el centro y sur del país, al mismo tiempo que generado aluviones en el altiplano del Norte de Chile (Romero et al., 2011a). Estos mismos años y por similares razones, intensas ondas de frío han cruzado de sur a norte el país (y gran parte de Sudamérica), produciendo heladas que han impactado la agricultura (la última de las cuáles se registró en Septiembre de 2013 causando pérdidas y efectos muy negativos sobre el empleo agrícola) así como cientos de víctimas entre los pobres urbanos (especialmente en las capitales de Argentina, Chile, Paraguay y en las ciudades principales del sur de Brasil) (RoMERO y MENDONÇA, 2011). En los veranos de los años 2012 y 2013, se registraron lluvias intensas en lugares y en una estación donde generalmente no ocurren por pertenecer a los climas de tipo mediterráneo, inundando pueblos y campos e interrumpiendo las vías de comunicación en el altiplano andino, además de producir inusuales precipitaciones sobre las altas montañas de la Cordillera de los Andes de Chile Central, causando aluviones de lodo que aumentaron la turbiedad de las aguas que abastecen las principales metrópolis, significando que en dos ocasiones, en el mes de enero y febrero de 2013, millones de habitantes de Santiago y de Valparaíso (las dos principales metrópolis chilenas, donde habita el $60 \%$ de la población nacional) debieron permanecer por días sin abastecimiento de agua potable.

Como se puede apreciar, pocas veces la sociedad chilena había percibido la alta exposición y vulnerabilidad ante las amenazas asociadas a eventos extremos de la naturaleza. Como es habitual, las explicaciones para estas catástrofes se han circunscrito a considerarlas como un producto de las amenazas naturales (CuTTER et al., 2003), destacando en especial sus causas geofísicas y meteorológicas, al mismo tiempo que se improvisaban imaginadas asociaciones con fenómenos tales como los cambios climáticos, o bien se ratificaba la fatalidad de pertenecer al "Círculo de fuego del Pacífico", sin que se expusieran en cada caso los antecedentes que permitían validar estas afirmaciones.

Sin embargo, no se ha discutido en Chile, con igual ponderación, la situación de vulnerabilidad social (ADGER, 2006), que presenta su sociedad, debido, en primer lugar, a la exposición (MC LAUGHIN y ARETZ, 2008) causada por su concentración espacial creciente sobre paisajes naturales amenazantes (Collins, 2009; Mustafa, 2005), que resultan principalmente de la extensión del país por zonas 
climáticas muy diferentes, que abarcan desde el Desierto de Atacama -uno de los más áridos del mundo- hasta los campos de hielos y glaciares permanentes de la Patagonia, todo ello junto a la omnipresencia de las altas pendientes que derivan del predominio de las montañas andinas y costeras y la presencia de innumerables arroyos y ríos, de caudales torrenciales debido a que la distancia que separa las altas cumbres andinas de sus desembocaduras en el mar promedia solo 150 $\mathrm{Km}$. A ello se debe agregar la existencia de múltiples volcanes activos y de innumerables fallas geológicas, enmarcados por el occidente por una línea de costa de aproximadamente $6.000 \mathrm{Km}$., ubicada sobre el Eje de Subducción de la Placa Sudamericana, una de las zonas de mayor frecuencia y magnitud de terremotos y tsunamis en el mundo.

La exposición permanente ante múltiples amenazas naturales no ha sido debidamente considerada por la legislación que regula la urbanización y construcción de viviendas e infraestructuras en este país, lo que se traduce especialmente en la ausencia o debilidad de los instrumentos de ordenamiento territorial, en general, y en la no incorporación en ellos de los riesgos y amenazas. Si fueran efectivos, indudablemente implicarían regulaciones que restringirían el uso y ocupaciones humanas de grandes extensiones del territorio, lo cual resulta incompatible con los intereses económicos que pretenden localizar sobre ellos urbanizaciones, carreteras, fábricas, campos agrícolas, explotaciones forestales, minas y túneles. Bajo tales restricciones naturales, el ordenamiento territorial solo podría emanar de acuerdos políticos entre los actores, impulsados por un Estado que brindara garantías de cientificismo, transparencia, respeto por el bien común, equidad y justicia, todo lo cual demanda un alto profesionalismo, una elevada capacidad de convocatoria y, esencialmente, una relevante legitimidad. Sin embargo, el desarrollo de Chile ha tenido lugar durante los últimos cuarenta años bajo un modelo neoliberal, que encontró en este país desde el primer momento una alta acogida por parte del gobierno conformado por una dictadura militar, encabezada por el general Pinochet y asesorada por destacados economistas de la Escuela de Chicago. Ello ha significado la reducción sistemática de la influencia y poder político del Estado, lo que ha resultado justamente en lo contrario de lo que era conceptualmente requerido por el ordenamiento territorial. El modelo neoliberal de la economía y la presencia en lo político de un Estado meramente subsidiario, se tradujo en una generalizada y persistente transformación de la totalidad de los componentes del territorio en bienes y servicios transables en el mercado, para lo cual fue imprescindible su "comodificación" y privatización, cuya administración ha sido entregada al mercado y con ello a los sectores de mayor poder hegemónico en la toma de decisiones, al mismo tiempo que se han desatendido cuestiones tan relevantes como la seguridad de los sectores mayoritarios ante la exposición a las amenazas naturales. La Constitución Política de 
Chile asegura el ejercicio del derecho de propiedad privada sobre componentes territoriales como el agua, el aire, los suelos y terrenos y la biodiversidad, para lo cual es necesario el funcionamiento de mercados libres y la determinación de precios por la oferta y demanda de los mismos. La ocupación urbana ilimitada ha cubierto con construcciones y sin mayores consideraciones respecto a sus restricciones y riesgos, áreas y sitios que nunca debieron haber sido urbanizados, y por el contrario, deberían haber sido destinados a la protección de la sociedad y la naturaleza, bajo una organización administrativa y jurídica que asegurara la función social de la propiedad, y que evaluara no solo el valor comercial de los terrenos o de sus bienes y servicios, sino que también el social, ecológico y ambiental. (Vidal y Romero, 2010; Romero y Vidal, 2013).

En Chile no existe Ley del Suelo ni de Ordenamiento Territorial, como tampoco manejo integrado de cuencas, planificación ecológica ni medioambiental y la planificación espacial y territorial ha sido estigmatizada como práctica centralista, contraria al libre mercado, y todas ellas como intervenciones estatales arbitrarias y burocráticas, obstaculizadoras para conseguir y mantener altas tasas de inversión privada y crecimiento económico. La protección de la sociedad ante amenazas naturales está entregada a una dependencia de menor nivel jerárquico y solo concentrada en atender funciones de emergencia y de post-desastre.

Por otro lado, se debe considerar también la existencia de una sensibilidad social creciente (Mc Laughin y Aretz, 2008; Cutter et al., 2003; Cutter y EmeRICH, 2006), que junto a la exposición, determina parte importante de la vulnerabilidad social, proceso por el cual finalmente las amenazas naturales se transforman en riesgos y eventuales desastres. En Chile, la sensibilidad social es mayor y se encuentra concentrada en los grupos sociales de menores ingresos económicos (la clase media baja y los pobres alcanzaban al $63,5 \%$ de la población nacional en el año 2012), que reside sobre áreas de amenazas naturales o medio ambientes degradados, empujados por las necesidades de vivienda e infraestructura no satisfechas de buena manera por las políticas, instituciones e inversiones públicas.

Por último, en realidad no se ha dispuesto de políticas públicas destinadas a generar y fortalecer la resiliencia de las comunidades ante la ocurrencia de amenazas naturales y sociales, que deberían formar parte de procesos de preparación para su enfrentamiento y recuperación. Ello se ha debido, en primer lugar, al interés por desconocer todo acontecimiento que altere la plusvalía de los terrenos o que intente regular el ejercicio del libre mercado como principal asignador de recursos. En segundo lugar, a la persistencia de la pobreza y segregación socioespacial y socioambiental de grupos mayoritarios de población urbana y rural, que se explica a su vez, en gran medida, tanto por déficits estructurales de los servi- 
cios públicos de calidad, especialmente en el área de salud y educación, como por la falta de objetivos de equidad en la distribución de éstos y de los equipamientos e infraestructuras públicos.

De esta forma, si los desastres ocurridos en Chile fuesen considerados como socionaturales y no solamente como fenómenos físicos (CUTTER et al., 2003; Collins, 2009), sería necesario reconocer, en primer lugar, el predominio de una visión "fisicalista" que se expresa en la existencia de una evidente asimetría en los conocimientos científicos disponibles sobre el comportamiento de las amenazas naturales en comparación con aquellos sobre vulnerabilidad social, lo que equivale a contrastar la cantidad y calidad de los estudios existentes sobre la naturaleza respecto a los que tratan la sociedad, con evidente desequilibrio en favor de los primeros (DAuPhiné y Provitolo, 2013). Adicionalmente, se aprecia la fragmentación de los conocimientos entre las ciencias naturales y las ciencias sociales, que en el caso de la geografía encuentra su correlato en la separación entre la geografía física y la social, enfoques que en gran medida, constituyen un factor explicativo sobre las limitaciones del actual conocimiento disponible para la formulación de políticas públicas para enfrentar los desastres socionaturales (Dauphiné y Provitolo, 2013; Demeritt, 2009). La propia naturaleza de los conocimientos científicos y las actuaciones públicas y privadas que sostiene se convierten en parte significativa de la generación de la vulnerabilidad social de las comunidades locales, como los han demostrado los desastres que se comentan en este trabajo (Romero y Mendoza. 2011, Albornoz et al., 2013; Albornoz y ROMERO, 2013)

Es evidente que se requiere de esfuerzos muy relevantes para conseguir superar la actual falta de conocimientos científicos sobre los desastres socionaturales y participar activamente en la formulación de políticas públicas para la preparación, enfrentamiento y recuperación de las poblaciones afectadas. Ello implica, por un lado, la existencia de una vocación interdisciplinaria y una perspectiva compleja de análisis, que reconozca a los desastres como propiedades emergentes de los sistemas territoriales. Por otro lado, es necesario desarrollar la ciencia de los desastres socionaturales como una disciplina propia, que sea capaz de integrar los aportes más significativos de las ciencias sociales y naturales, no como externalidades, sino como interferencias fundamentales (DEMERITt, 2009). Adicionalmente, disponer de una actitud de diálogo con los diferentes actores que participan en la construcción de la vulnerabilidad social y una capacidad de análisis y comprensión de sus factores económicos, sociales, culturales y políticos (Cutter y EMerich, 2006, Adger, 2006). 
En Chile, muchos de los estudios sociales publicados recientemente respecto a los desastres "naturales" no poseen carácter científico, sino que mayoritariamente se trata de literatura "gris" (sin revisión de pares), consistente en informes de consultorías contratadas por el gobierno o bien provenientes de organismos públicos destinados a legitimar sus actuaciones ante la emergencia o a publicitar su autodeclarada eficiencia ante la reconstrucción. Algunos resultados de investigaciones académicas se presentan como prácticas profesionales, memorias de pregrado y excepcionalmente como tesis de postgrado, además de la publicación de algunos libros, carentes de comités editoriales. No ha existido tampoco un debate en el seno de las ciencias sociales respecto a la necesaria focalización de sus investigaciones, por lo que tienden a predominar estudios introductorios sobre diferentes tópicos. En estos aportes se aprecia una urgencia por participar en intervenciones y aplicaciones del conocimiento, que no necesariamente responde a los resultados de las investigaciones científicas. La gestión tiende a remplazar a la investigación, lo que impide la generación sistemática de conocimientos que permitan establecer las principales causalidades de los fenómenos, su comparación con los marcos de referencia aportados por la literatura internacional y su relación con fundamentos teóricos y procedimientos metodológicos apropiados.

Los estudios de casos, consistentes muchas veces en verdaderas monografías de situaciones únicas o de visiones ideologizadas no han favorecido la disponibilidad de conocimientos útiles, siendo notable la carencia de informaciones y análisis sobre cuestiones tales como el capital y los bienes sociales y el rol jugado por las redes y las instituciones comunitarias, vecinales y familiares en cada uno de los eventos. Los análisis no consideran con la debida profundidad los antagonismos, luchas de poder y conflictos que se generan entre los actores, especialmente por apropiarse y controlar los territorios y sus recursos, como tampoco permiten explicar situaciones de vandalismo y saqueos que han acompañado a estos eventos en Chile y otros países. Las participaciones socio políticas no son mencionadas, por lo que actuaciones evidentemente inapropiadas por parte de los actores y las instituciones son ignoradas, con lo que se obstaculiza su perfeccionamiento ${ }^{2}$. El clientelismo político remplaza las asignaciones de ayudas e

2 En este sentido es absolutamente inédito que la Corte Suprema haya condenado por primera vez al Fisco a pagar una indemnización de 55 millones de pesos chilenos (aproximadamente US\$ 100.000) por la muerte de Mario Ovando, que falleció ahogado por las olas del tsunami del 27 de febrero de 2010 en la ciudad de Talcahuano, obligando de esta forma al Estado a asumir una "falta de servicio" en la medida que la autoridad regional informó erróneamente de que no había peligro de que ello ocurriera y, por lo tanto, no debían evacuar sus hogares. La condena "por daño moral" indica que "lo vivenciado por los demandantes (...) ha debido generar una merma en el patrimonio espiritual de quienes saben que, de no haber mediado la infeliz falta de servicio, no habrían debido soportar la carga que ahora conducen" (El Mercurio, 20 de diciembre de 2013, C 
inversiones en forma justa, equitativa y proporcional a los niveles de vulnerabilidad social de los diversos grupos. Se advierte todo tipo de improvisaciones y descoordinaciones institucionales y un afanado empeño por impedir la actuaciones de las organizaciones sociales de base, predominando en todos los casos las decisiones adoptadas autocráticamente desde arriba hacia abajo (Albornoz y Romero, 2013). En muchos casos, argumentando la urgencia de las respuestas, se evita incluso comunicar los planes a las poblaciones involucradas, las que consecuentemente viven momentos de angustia, incertidumbre y desconfianza, lo que termina debilitando un capital social escasamente desarrollado (ADGER, 2003; Cutter y Emerich, 2006). Se conoce muy poco sobre los rasgos culturales, topofilia, identidad, pertenencia y arraigo entre la población y sus lugares de residencia, que se manifiestan siempre como rechazo y resistencia frente a los traslados y evacuaciones ordenados por la autoridad, muchas veces en forma apresurada, improvisada y equivocada.

La atención a la emergencia y la concentración exclusivamente en la reconstrucción física de los lugares, relega las obligaciones públicas frente a los procesos de recuperación sicológica, económica, social y cultural. No existen políticas públicas destinadas a preparar la población ante desastres o bien éstas se reducen a simulacros o planes de educación formal que no se relacionan con la vulnerabilidad antecedente de los sistemas sociales, construidos y naturales de los lugares (CUTTER et al., 2008). Las etapas de recuperación y reconstrucción son asumidas por las autoridades de turno, como una prueba de su eficiencia política, esforzándose por demostrar que han sido capaces de resolver todas las dificultades en el más breve plazo, mostrando abultadas y controvertibles estadísticas y rechazando cualquier crítica de carácter académico, lo que se estima superfluo e inoportuno. Las soluciones post-desastre se restringen principalmente a construcciones físicas, no forman parte de planes integrales de desarrollo y no aprovechan las lecciones aprendidas por las sociedades locales. Se desconoce el hecho de que en todos los casos, son las redes de las organizaciones locales, lideradas por vecinos de plena confianza y sustentadas por las organizaciones sociales de base, actuando en forma coordinada con los representantes de los servicios públicos, lo que ha garantizado la resiliencia y supervivencia de comunidades, fortalecido

12). Juristas a favor de la decisión sostienen que "la falta de servicio se explica por una extrema ineptitud de todos los órganos de la administración del Estado para reaccionar frente a un evento que en Chile es por completo previsible" (Enrique Barros, profesor de la Universidad de Chile en El Mercurio, 20 de diciembre de 2013, C13). Por el contrario, quienes discrepan afirman que se trató "de un daño que proviene de un caso fortuito frente al que no puede imputarse responsabilidad de ningún tipo (...) y que no puede ser indemnizado. Se trataría de lo que el derecho anglosajón denomina el mal que nos envía el buen Dios" (Pedro Pablo Vergara, El Mercurio, 20 de diciembre de 2013, C13). 
su capacidad de absorción de las amenazas y facilitado su recuperación en forma rápida y eficiente. En vez de proponer una reestructuración sociopolítica y territorial, basada en instituciones autónomas y descentralizadas, que den cuenta de la realidad de los lugares, se insiste en generar organismos centralizados, jerarquizados y autorreferentes, que han demostrado una y otra vez y en todas las ocasiones ser los primeros en resultar aislados del resto de la comunidad.

Es necesario apreciar la importancia de la existencia y fortaleza del capital social, de sus bienes, organizaciones e instituciones, así como del aprendizaje social, para la generación y fortalecimiento de mecanismos y acciones de resiliencia territorial (AdGER, 2003; CUTTER y EMERICH, 2006), lo que ha permitido no sólo reducir las pérdidas, sino que además transformar en muchos casos a estos eventos adversos en oportunidades de movilización social y política, que deben apoyarse, tanto para asegurar un mejor desempeño de la sociedad ante futuros sucesos, como para integrarla en las acciones de recuperación y reconstrucción en proyectos amplios de desarrollo sustentable, incluyendo sus componentes económicos, sociales y ambientales. Es evidente que resulta imposible esperar que el mercado se haga cargo de los planes de prevención, preparación, educación, capacidad de absorción, resiliencia y aprendizaje social que se requiere para evitar que sigan aumentando los efectos de los desastres naturales que agobian a Latinoamérica. Solo adecuados y eficientes procesos de ordenamiento territorial, generados y controlados por la sociedad, permitirán que al mismo tiempo se encuentren localizaciones seguras para los sectores sociales vulnerables y se impida la ocupación de terrenos cuya historia ambiental permite clasificarlos como riesgosos y amenazantes. Para ello, como para implementar las diferentes etapas, antes, durante y después de ocurridas las amenazas naturales, se requiere de instituciones públicas técnica y profesionalmente capacitadas, dotadas de suficiente poder político y sostenida por las demandas y participaciones, propias de una sociedad genuinamente democrática, justa y educada.

\section{METODOLOGÍA}

"Mientras la vulnerabilidad física puede ser fácilmente identificada usando datos de eventos pasados, los aspectos sociales de la vulnerabilidad a las amenazas son un poco más complicados dada su variabilidad temporal y espacial (CUTTER y EMERICH, 2006, p. 104) 
La geografía ambiental (CAStree et al., 2009; Demeritt, 2009; Romero et al., 2011b) puede cooperar enormemente a una renovada comprensión de los desastres socionaturales, en la medida que reconoce tanto la importancia de contar con las mejores informaciones y explicaciones especializadas proporcionadas por las disciplinas que conforman la geografía humana y la geografía física, como la necesidad de conseguir síntesis e integraciones que den cuenta de propiedades emergentes, entre las cuales se encuentran las amenazas naturales y vulnerabilidades sociales cuya superposición e interacción territorial permite predecir y explicar la ocurrencia de los desastres (CUTTER et al, 2008). En el caso del terremoto y tsunami que destruyó gran parte de Chile Central, ha sido necesario utilizar sistemas de información geográfica para percibir la manera en que se fue construyendo la exposición de los sectores socialmente más vulnerables, consistente en la urbanización sistemática, ordenada por el Estado o por las urgencias sociales, de áreas y sitios que presentaban una historia de amenazas naturales, superponiendo espacialmente inundaciones causadas por lluvias intensas, olas de tsunamis y anegamientos de suelos (Romero y VIDAL, 2014; Romero et al., 2010 y 2011b). Al examinar espacialmente la ocupación de áreas riesgosas por parte de la población socialmente más vulnerable durante los últimos cincuenta años, se aprecia, mediante fotointerpretación y análisis de imágenes satelitales, como se fue construyendo gradualmente la exposición territorial, sin que los instrumentos de ordenación del territorio dieran cuenta de ello, hasta culminar con el arrasamiento de extensas zonas por el tsunami de 2010 o bien su inundación en las lluvias extraordinariamente abundantes de 2005 y 2006 (VIDAL y RoMERO, 2010). Se trata en general de las mismas áreas, que son afectadas por amenazas en forma recurrente, sin que se aprecie una respuesta institucional pertinente, tal como impedir su urbanización, declarar tales áreas como de protección social o destinarlas a la conservación de la naturaleza. Aún más, los planes públicos que regulan el uso de los territorios omitieron sistemáticamente la consideración de la información científica, elaborada por los propios organismos del Estado, incentivando en muchos casos la ocupación de tales territorios riesgosos que fueron asignados a usos residenciales o industriales (ROMERo et al., 2010; Romero y VIDAL, 2013).

El análisis de los acontecimientos sucedidos durante la noche y madrugada del 27 de febrero de 2010, que es posible de reconstruir a través de reportajes periodísticos, pero especialmente de los textos judiciales e informes de peritos policiales, permite concluir que la totalidad de los organismos del Estado no contó con las informaciones ni sistemas de comunicación que le permitieran alertar a la población sobre la asociación entre la magnitud 8,8 del terremoto y la ocurrencia de ondas de tsunami que alcanzaron hasta 40 metros de altura y se registraron en las horas siguientes a las 3,34 horas de la madrugada, en que se registró el sismo (Romero et al., 2011b). Ciudades y pueblos costeros fueron cubiertos por las olas 
causando numerosas víctimas, que se pudieron haber evitado si el Estado hubiese contado con el personal capacitado, la información adecuada y oportuna y sistemas de comunicación con la población. El desentendimiento de la calidad profesional, académica y tecnológica de los servicios públicos por décadas, junto a la privatización de funciones esenciales, como las comunicaciones, generó un caos institucional que el país desconocía y que aún es objeto de análisis y discusiones.

Sin embargo, durante todo este tiempo han primado las acusaciones políticas entre los partidos del gobierno y la oposición, sin que se produzca el debate necesario ni se disponga de análisis profundos sobre las carencias institucionales, así como sobre la falta de preparación de la población. Muchas áreas no sólo fueron devastadas por las olas o las ondas sísmicas, sino que a continuación fueron objeto de saqueos y vandalismos, lo que indica actuaciones sociales que seguramente denuncian oportunismos, resentimientos profundos y revanchismos, en gran parte producto de las desigualdades, de una sensación de abuso por parte de la población respecto a los comerciantes y distribuidores, y de una localización desaprensiva que localizó en las vecindades grupos sociales socioeconómicamente contrastados sin mediar ningún esfuerzo de integración real. Ello no debe, sin embargo, ocultar las numerosas lecciones brindadas por personas e instituciones locales que asumieron desde el primer momento, actuaciones plenas de solidaridad, reciprocidad y protección social de las comunidades, que deben ser reconocidas, sistematizadas y utilizadas para ofertar nuevas organizaciones sociopolíticas, de carácter esencialmente local y que se alejan considerablemente de las actuaciones centralizadas, herméticas y descoordinadas que se observó por parte de actores públicos y privados.

Las entrevistas practicadas a representantes de diversas comunidades locales afectadas indican con claridad el rol del capital y los bienes sociales en las etapas cruciales de enfrentar la emergencia, los efectos posteriores y la reconstrucción (ECO-Universidad de Concepción, 2011; Romero y Mendonça, 2012). Nuevamente, se aprecia que hay muchos más aportes de los geofísicos y diversos especialistas en ciencias de la tierra, que de los especialistas de las ciencias sociales, lo que podría implicar que no se hayan sistematizado numerosas actuaciones que en muchos casos significaron la salvación de miles de personas. Los relatos indican cómo funcionarios de la Armada, Carabineros (policía), y bomberos (servicio formado por vecinos que actúan en forma voluntaria), organizaron evacuaciones de poblaciones (como en el caso de Pichilemu), sin esperar órdenes externas y sobre la base de sus propias observaciones de evidencias aprendidas intergeneracionalmente y mediante memorias, diálogos familiares y sus propias experiencias personales. Es necesario que la geografía insista en el estudio multiescalar y multidisciplinario que caracteriza a las decisiones que se adoptan 
antes, durante y con posterioridad a la ocurrencia de desastres socionaturales, superando tanto las barreras que impiden apreciar los complementos o contradicciones entre las actuaciones y decisiones que se adoptan a escalas regionales, subregionales, locales, familiares e individuales, como proporcionando marco de referencia generales y contextualmente específicos y propios de los lugares.

La investigación geográfica no puede renunciar a la producción, interpretación e integración de los conocimientos espaciales respecto al comportamiento de las variables que caracterizan la extensión, magnitud y frecuencia de las amenazas naturales, debiendo ser capaz de generar los niveles de informaciones específicas y sus respectivos modelos de integración. Las áreas afectadas por terremotos, aluviones, tsunamis y erupciones volcánicas son las mismas y han sido devastadas en varias ocasiones en casos examinados en diversos lugares de Chile. De la misma manera, diversos niveles de información socioeconómica, contribuirán a incluir aspectos de la vulnerabilidad social en la generación de modelos integrados de riesgos. No obstante, el mayor desafío de encuentra en la incorporación y desarrollo de los métodos cualitativos, cuya fuente de información son los propios actores locales. No cabe duda que las ciencias sociales, en especial la sicología y antropología, disponen de mucha experiencia para contribuir a explicar los comportamientos humanos ante las amenazas naturales y la geografía se puede beneficiar grandemente del trabajo conjunto. Otro tanto debería suceder con los métodos aplicados por las ciencias políticas y en especial, por la ecología política y las ciencias de estudio de la vulnerabilidad social y recuperación ante desastres, todas las cuáles exhiben una amplia experiencia a nivel mundial. En nuestro caso, la aplicación de numerosas entrevistas a los habitantes de zonas afectadas por terremotos, tsunamis y aluviones, así como la consideración de relatos reproducidos en diversos medios, nos ha conducido a reconocer la importancia de los discursos expresados por los diversos actores y la forma en que interpretan los eventos (Mustafá, 2005), sobresaliendo los intereses políticos, sea para denunciar injusticias, reclamar ante discriminaciones, justificar actuaciones de la autoridad o legitimar decisiones discutibles. De mayor complejidad ha resultado, sin embargo, la transformación de interpretaciones subjetivas en categorías de análisis social, que finalmente permitan explicar las actuaciones colectivas de las comunidades locales y la manera en que dan cuenta de la complejidad de las relaciones entre la naturaleza y la sociedad.

\section{RESULTADOS Y CONCLUSIONES}

"Las vulnerabilidades sociales preexistentes dan origen a la catástrofe social: las 
amenazas morales ocurren por nuestra inhabilidad colectiva para responder adecuadamente" (CUTTER y EMERICH, 2006, 106)

El tema de la multiescalaridad ha permitido interpretar la ocurrencia de aluviones en las alturas de las montañas andinas en el Norte y Centro de Chile y ha hecho posible comprender las interacciones entre índices climáticos que permiten explicar la ocurrencia de lluvias y nevazones en los mismos bordes del Desierto de Atacama o en el verano de Chile Central (Romero et al, 2011 a). La ocurrencia simultánea de la fase negativa de la Oscilación Decadal del Pacífico y de la Oscilación del Sur del Pacífico (La Niña), junto a una inusual fortaleza y alcance de la Convergencia Antártica, permite explicar, además de las lluvias excepcionales y los aluviones de lodo y barro que arrasaron campos de cultivos, aldeas y pueblos del Norte de Chile, la ocurrencia de ondas de frío y sequías, con sus consiguientes impactos sobre la agricultura y las fuentes de energía en el centro y sur del país. No obstante, el verdadero significado ambiental de estos eventos climáticos extremos, se observa en la escala topoclimática y especialmente en las cuencas andinas, donde los procesos convectivos y las rutas de nieve y lluvias son conducidas por la altura, exposición y coherencia de los relieves, sin que se cuente en la mayoría de los casos con información adecuada y se deba recurrir a modelos de gran escala que permanecen sin validarse por falta de estaciones de monitoreo.

Las variabilidades y extremos climáticos, actúan esencialmente sobre la disponibilidad de recursos hídricos de superficie y subterráneos, de los cuáles depende a su vez la supervivencia de ecosistemas y comunidades indígenas y rurales que viven en las montañas y sus valles anexos. Las estrategias de adaptación social frente a estos eventos, implican la existencia de instituciones y organizaciones que controlan extensos territorios, caracterizados por su diversidad de recursos y por ello, por una significativa resiliencia territorial frente a la escasez. Ecosistemas, humedales y cauces fluviales organizan pisos ecológicos "atados" (ZimMERER, 2003), circuitos y redes de asentamientos poblados desde tiempos prehistóricos, teniendo a la ganadería de auquénidos como recurso fundamental. El significado ontológico de la seguridad de estos lugares y su representación social y cultural (física y simbólica) (HAwKIns y MAURER, 2011), se encuentra, sin embargo, severamente amenazado por las empresas transnacionales que han adquirido o intentan obtener derechos de agua, empleando para su beneficio una legislación que desde 1981 transformó a este recurso en una comodity más, que se transa libremente en el mercado y cuyo precio depende exclusivamente de la ley de oferta y demanda (Romero et al., 2012). El debilitamiento de la resilien- 
cia territorial de las comunidades andinas, debido a la pérdida de propiedad y control de los recursos hídricos, ha implicado el abandono de tierras de cultivo y de circuitos de trashumancia ganadera, tornando redundante la mano de obra y forzando su migración hacia las ciudades y abandono de sus modos de vida tradicionales y en la aparente pérdida de control de la propiedad sobre sus recursos territoriales. Sin embargo, como en el caso de los procesos geográfico físicos, que necesitan de una visión multiescalar para ser comprendidos, en el caso de las actuaciones sociales es de similar importancia explorar de qué manera se imbrican dialécticamente los procesos provocados por las compañías transnacionales, los organismos e instituciones nacionales y las comunidades locales, siendo de primordial importancia apreciar las forma de negociación que se han establecido en los diversos lugares y sus profundas transformaciones sobre los flujos de personas, informaciones y recursos que en la actualidad definen las relaciones entre las tierras altas y bajas y entre los campos y las ciudades.

De la misma manera que se requiere practicar entrevistas e investigaciones participativas para conocer las razones y formas de actuación de los diversos actores, en los campos y ciudades, es necesario considerar los aprendizajes y lecciones, fuertemente dependiente de los contextos históricos, espaciales y culturales, que permiten evaluar cuidadosamente si se trata de experiencia de adaptación exitosas o no y de los mecanismos y procedimientos que han concurrido a desenvolver unos y otros. En realidad, las investigaciones de campo demuestran que aún entre comunidades vecinas se observan alianzas y comportamientos diferentes, al mismo tiempo que experiencias de traslocación que explican la interacción entre escalas locales y regionales mediante flujos de informaciones y bienes, tangibles e intangibles, que son vitales para la adopción de decisiones en favor de algunas comunidades locales y que en altiplano andino, por ejemplo, se remontan a organizaciones espaciales en muchos casos heredadas de tiempos prehispánicos. Si la resiliencia territorial constituye un componente fundamental de la reducción de la vulnerabilidad social de las poblaciones rurales, es muy importante considerar las relaciones naturaleza-sociedad que se concretan y transforman permanentemente al interior de las ciudades, caracterizadas en América Latina y en Chile , por profundos, persistentes y crecientes procesos de segregación e injusticia socioambiental. Frente a los terremotos y tsunamis que han tenido lugar en Chile es evidente que los más afectados han sido los sectores de menores ingresos, que, por un lado, han sido forzados a ocupar terrenos expuestos a amenazas naturales, que se han concretado, posteriormente como desastres, destruyendo sus viviendas y concentrando socialmente a las víctimas (ROMERo et al 2011b; ROMERO y VIDAL, 2013). Nuevamente, los SIG han permitido superponer espacialmente las áreas mayormente afectadas con la distribución de la población según sus niveles socioeconómicos, comprobándose que los estratos pertenecientes a las clases me- 
dias y medias bajas constituyen la mayoría. Por el contrario, los grupos sociales de altos ingresos económicos se localizan en lugares más seguros, disponen de construcciones robustas y sus planes de urbanización excluyen permanentemente las áreas de riesgos. Algo similar se ha observado en Chile respecto a la localización de la población en áreas expuestas a inundaciones, aluviones y remoción en masa de sedimentos, especialmente en los piedemontes de las montañas andinas y costeras que rodean las metrópolis de Santiago, Valparaíso y Concepción y que resultan comparables con otras situaciones de desastres urbanos observados en Brasil, por ejemplo (Romero et al., 2009; Romero y MENDONÇA, 2012). En todos estos casos, es claro que no solo se somete a los grupos sociales más vulnerables a ocupar paisajes amenazantes, sino que además la precariedad de sus viviendas e infraestructura pública, acentúan sus riesgos de manera desproporcionada, en una evidente demostración de falta de justicia ambiental. Lo que resulta especialmente preocupante es la reiteración y persistencia de estos desastres socionaturales, así como su aumento en frecuencia y magnitud en la medida que la población más vulnerable se concentra espacialmente sobre áreas amenazantes, sin que se implementen políticas y planes públicos eficientes. Para explicar esto, es necesario explorar en profundidad los discursos de los diferentes actores, evitando los argumentos simplistas que recurren a los cambios climáticos o a una supuesta porfía de los grupos afectados por volver a habitar sitios amenazantes (HIROYUKI, 2012).

Por otro lado, en la mayoría de los actores entrevistados en las zonas urbanas periféricas afectadas por terremoto, tsunami e inundaciones en Chile y Brasil (Romero y MENDONÇA, 2012), predominan los relatos que se inician con ocupaciones ilegales de terrenos no ocupados debido a su condición amenazante o bien por estar bajo especulación urbana. Debido a las luchas por conseguir resolver sus urgencias sociales de vivienda y equipamiento urbano, las organizaciones vecinales consiguieron la regularización de la propiedad de la tierra y procedieron a realizar procesos de autoconstrucción, muchas veces con materiales precarios sobre áreas riesgosas, donde sus familias se asentaron y extendieron, aumentando el número de personas y hogares y de esta forma, la sensibilidad de la población expuesta. Una vez ocurrido el desastre, estos grupos han conseguido mediante sus luchas, la realización de inversiones estatales o privadas que han implicado su traslado a áreas más seguras o bien el mejoramiento y aseguramiento de sus viviendas, con lo cual es posible apreciar que la tragedia se transformó en oportunidad sobre la base de la calidad de su capital y bienes sociales. No obstante, en muchas ocasiones, la reconstrucción sobre sitios inaptos o riesgosos no ha logrado sino aumentar la vulnerabilidad ante futuros eventos.

La falta de atención a los aspectos sociales resulta dramática en el caso de la erupción del Volcán Chaitén, que afectó a la ciudad del mismo nombre, en el 
sur de Chile, desde el 1 de mayo del año 2008 y que significó la evacuación total y obligatoria de más de 5.000 habitantes. En este caso, el gobierno contrató dos importantes consultorías: la primera en los meses inmediatamente posteriores, con la finalidad de adoptar la decisión de reconstruir la ciudad en el mismo sitio originario, o de lo contrario, trasladarla a uno cercano más seguro. Para ello recurrió a dos universidades privadas (PUC y UACH, 2009), que se concentraron especialmente en demostrar que el terreno donde se encontraba la ciudad con anterioridad a la erupción concentraba una serie de amenazas naturales y que por ello, era necesario no sólo ubicar con urgencia un sitio para su refundación, sino que además iniciar lo más pronto posible la instalación de los servicios públicos que atrajeran a la población evacuada y, especialmente, impidiera el retorno de los habitantes hacia el lugar originario, con el cual mantenías relaciones de pertenencia, arraigo e identidad, en términos sociales, culturales y económicos.

Una segunda consultoría (SUBDERE, 2012), finalizada a comienzos de 2012, contratada a una empresa privada especializada, tenía por objetivo evaluar las probabilidades de ocurrencia y los alcances espaciales de un conjunto de amenazas naturales que se desencadenaron con motivo de la erupción del Volcán Chaitén (flujos piroclásticos, nubes de cenizas, avalanchas de lahares, aluviones e inundaciones, remoción en masa). Los SIGs resultaron especialmente útiles para explicar y comprender áreas bajo distintos grados de amenazas por los distintos riesgos naturales y para conseguir su integración espacial para generar mapas de la vulnerabilidad física que presentan los diferentes centros poblados que se localizan en el área de influencia, no sólo de las acciones del vulcanismo registrado en esta ocasión, sino que también de la eventual erupción de otros conos cercanos, activación de áreas generadoras de remoción en masa y eventual ocurrencia de terremotos y tsunamis, sobre los cuáles no se tienen registros históricos.

Ambas consultorías se concentraron exclusivamente en las amenazas naturales, obedeciendo demandas de conocimiento provenientes de los servicios públicos, lo que resulta inexplicable considerando la abundancia de experiencias y análisis científicos sobre la vulnerabilidad social como componente fundamental de los desastres. Los estudios mencionados definen la vulnerabilidad territorial solo respecto al alcance espacial y temporal de los fenómenos naturales seleccionados, aunque en el discurso reconocen la primordial importancia de considerar los estudios de vulnerabilidad social, sobre los cuáles no avanzan mayormente.

Sin disponer de memoria social ni informaciones científicas sobre erupciones previas ni sobre riesgos asociados a este volcán y ante la inminente amenaza de la ocurrencia de lahares que arrasaran el asentamiento poblado o el arribo de flujos piroclásticos a lo largo del valle que vincula a la montaña con la plataforma 
litoral donde se encontraba la ciudad, la autoridad ordenó la evacuación total de sus habitantes y la prohibición de regresar a ella, para lo cual procedió a instalarlos provisoriamente en pueblos y ciudades regionales, alejadas del epicentro, destinando recursos para su mantención y subsidios de sobrevivencia, que se prolongaron por un par de años, dejando de manifiesto una auténtica preocupación del gobierno, reconocida en general por los afectados y ampliamente publicitada con fines políticos. Si bien ambos fenómenos geofísicos finalmente no se manifestaron, la llegada de las lluvias intensas del invierno se encontró con los cauces fluviales colmatados por las cenizas lo que provocó el desborde de sus aguas, parte de las cuáles atravesaron la ciudad, arrasando con un sector importante de sus puentes, calles, y viviendas. Ello permitió validar la decisión política de evacuar a la población e impedir su regreso definitivo y generó una sensación de éxito de la operación, que a su vez, contribuyó a desplegar un imaginario nacional extraordinariamente optimista, que impidió ver la complejidad del asunto, su desarrollo en el largo plazo, sus implicancias multidisciplinarias y multiescalares, y alentó la búsqueda de soluciones rápidas, entre las cuáles se contaba la construcción de una ciudad nueva, en la localidad vecina de Santa Bárbara, que ofrecería una suma incalculable de ventajas en los ámbitos ecológicos y económicos y naturalmente, una seguridad infinitamente mayor frente a las amenazas naturales (lo que resultaba incomprensible en la medida que se ubicaba a solo $10 \mathrm{~km}$. de la ciudad destruida). Lo más relevante además, era que estaría completamente habilitada en el transcurso del año 2012.

No existen hasta el momento resultados de investigaciones en el ámbito de las ciencias sociales, que permitan conocer y evaluar la totalidad y complejidad del traslado masivo de población desde la ciudad de Chaitén, su instalación provisoria (o definitiva) en pueblos y ciudades cercanas, el retorno de una parte importante de la población a la ciudad de origen y el abandono de las iniciativas de reconstrucción de un nuevo asentamiento. En este sentido, resultan muy valiosos la memoria elaborada por MARChANT (2010) y la ponencia de SALGADO y UgARTE (2012). Ambos trabajos, realizados a partir del análisis de entrevistas a actores clave permiten describir la complejidad del proceso de traslado, sin que se contara con planes específicos de mediano y largo plazo ni tampoco con organismos públicos ni legislaciones que permitieran ejecutar el conjunto de acciones de evacuación, mantenimiento en los lugares de asentamiento transitorio, inserción social, económica y cultural de los trasladados, evitar efectos sicológicos negativos del desarraigo, precisión sobre el lugar y fecha de retorno, etc. Sin embargo, se trata de investigaciones exploratorias, que abarcan una gran variedad de relaciones y fenómenos, para cuya comprobación y análisis se requeriría contar con muestras representativas de los actores, sus lugares de origen y residencia temporal, además de sus características socio económicas, junto a adecuados marcos 
teóricos de interpretación. Los datos presentados permiten ratificar la importancia de los componentes socioeconómicos en la construcción de la vulnerabilidad, por sobre reacciones individuales, reflejada, por ejemplo, en el hecho de que los erradicados con mayor disponibilidad y acceso a los recursos, se establecieron en las ciudades principales, insertándose con mayor éxito en la complejidad de la vida urbana y beneficiándose de las redes de servicios públicos, mientras que los sectores más vulnerables, tendieron a hacerlo en pueblos pequeños, bajo el amparo sustancial de las redes familiares y practicando la continuidad de sus actividades laborales, concentradas especialmente en pesca y silvicultura.

Las investigaciones mencionadas son fecundas en relevar las relaciones espaciales y territoriales que, en forma idealizada o real, han sido relatadas por los actores y que supuestamente, vinculaban de manera especialmente armoniosa a los habitantes de Chaitén, entre ellos y con sus lugares de residencia, correspondientes a paisajes caracterizados por gran belleza natural, donde compartían modos de vida, seguridad y tranquilidad. Todos estos valores altamente positivos otorgados a sus lugares de origen eran contradictorios con las escasas cualidades reconocidas a los lugares donde fueron forzados a residir después de la evacuación. Esta reacción negativa permite explicar, por un lado, la insatisfacción general con los procedimientos implementados para su atención por el Estado, no obstante que les fueron otorgadas significativas ayudas económicas, albergues para enfrentar la emergencia, bonos para arrendar viviendas, dinero para la manutención de las familias y becas para los estudiantes, además de contención sicológica. Es evidente que los beneficios económicos no alcanzan a compensar las pérdidas asociadas a la ontología de los lugares ni a las deficiencias morales con que se enfrentan los desastres socionaturales,

En gran medida, la angustia e incertidumbre manifestada por los trasladados ha estado relacionada con la desarticulación de sus redes y tejidos sociales, causada tanto por la dispersión espacial de la comunidad en diferentes pueblos y ciudades (realizada sin mayor consulta a los habitantes, y ejecutada en forma desordenada, al punto de que no se conocía el destino de las embarcaciones, no se respetaron las listas de pasajeros ni se registraron sus nombres y números telefónicos como para ubicarles posteriormente) (MARCHANT, 2010), como por las dificultades de insertarse en nuevos modos de vida, más urbanos, y que abarcaron la totalidad de sus componentes, incluyendo subsistencia, alimentación, vivienda, educación, trabajo, relaciones sociales, exposición a una nueva cultura (SALGADO y UGARTE, 2012).

Adicionalmente, las actuaciones y los momentos políticos participaron activamente en la construcción y ampliación de la vulnerabilidad social de los habi- 
tantes de Chaitén, particularmente en la angustia e incertidumbre propiciada por contradicciones en los discursos del gobierno central, que se vieron agravadas porque a fines del año 2009 fue electo democráticamente un gobierno de signo políticamente diferente al que había dirigido el enfrentamiento del desastre. En Chile, los cambios de gobierno implican retrasos en las decisiones, desconocimiento de compromisos asumidos, reemplazo de jefes de servicios públicos (y de funcionarios que juegan roles clave) $\mathrm{y}$, en este caso particular, un cambio drástico de tipo ideológico desde un gobierno que apreciaba de mejor forma el rol de las actuaciones públicas a uno que, por el contrario, consideraba principalmente el rol del sector privado y las decisiones fundamentadas en el mercado. De esta forma, a comienzos de 2009, el gobierno que posteriormente sería sustituido, anunció que, de acuerdo a los estudios y análisis de riesgos practicados, era imposible pensar en re habitar la ciudad de Chaitén, lo que fue anunciado por el Ministro del Interior el 17 de febrero de ese año, comunicando a los desplazados, que deberían esperar en sus sitios de residencia temporal, el retorno a una nueva ciudad, denominada Nueva Chaitén, que se construiría a $11 \mathrm{Km}$. al norte de la destruida. El 10 de abril de 2009 se inician apresuradamente las obras de habilitación de la nueva ciudad, con la instalación provisoria del municipio, dependencias de policía y una pista de aterrizaje de emergencia, en la localidad de Santa Bárbara. En Marzo de 2010, las autoridades salientes hacen entrega de un Plan Maestro de habilitación de servicios públicos en la nueva ciudad (PROTEGe, 2009 y 2010), lo que alienta aún más a los desplazados a pronunciarse favorablemente para iniciar prontamente un retorno al nuevo asentamiento, aunque lo condicionan a la existencia de un conjunto de facilidades, que incluyen escuelas, puestos de salud y trabajos, especialmente en el sector turístico (Presidencia de la República, 2009). El gobierno presentó planos, maquetas y representaciones de la nueva ciudad, que corresponden a un diseño idealizado de relaciones ecológicas, económicas y sociales, mientras que para fundamentar sus propuestas arquitectónicas y paisajísticas, recurrió a fotografías tomadas especialmente de los países nórdicos, destacando Suecia y Noruega. El nuevo gobierno hizo suyas las decisiones adoptadas y el 25 de mayo de 2010, el Presidente ratificó el abandono de la antigua ciudad y la construcción de una nueva.

Sin embargo, en el mes de noviembre de 2009 el Gobierno Regional, y el 9 de diciembre de 2010 el mismo Presidente de la República, anunciaron que se dejaba de lado definitivamente la habilitación de la nueva ciudad y que se había decidido reconstruirla sobre el sitio original, aunque sólo en su sección norte, que presenta mayor seguridad ante las amenazas naturales y que no fue devastada por los acontecimientos de mayo de 2008. En abril de 2011 se oficializa esta decisión, anunciándose que se refundará la ciudad de Chaitén, iniciándose la instalación de los servicios básicos de luz y agua, solicitándose un estudio que aclarase el 
riesgo asociado a la inhalación de cenizas volcánicas por parte de la población y la ejecución de obras de mitigación de las amenazas naturales, limpieza de la zona, protección de la ribera del río, arborización del sector sur (arrasado por las inundaciones) y habilitación de una pista de aterrizaje. En enero de 2013 se informó que el nuevo aeropuerto, ubicado en la localidad de Chana, a $22 \mathrm{Km}$. de Chaitén, estaría completamente habilitado en el mes de enero de 2014, lo que, desde luego, no sucedió.

Como en febrero de 2012 se dio a conocer el informe de consultoría contratado para establecer y clasificar las probabilidades de riesgos y vulnerabilidades físicas que presentan las principales localidades urbanas de Chaitén (SUBDERE, 2012), el 1 de junio de este último año, mediante un documento oficial enviado a los secretarios regionales de los ministerios de Obras Públicas y de Bienes Nacionales, el Secretario Regional del Ministerio de Vivienda y Urbanismo, comunica que "Chaitén completo, es un territorio vulnerable frente a riesgos naturales, por lo que su ocupación siempre conllevará un riesgo para las personas que lo habiten o realicen actividades en él y también se arriesga la pérdida o el serio deterioro de la infraestructura localizada ahí" (cit. MACAYA, 2012). Esta misma autora se pregunta sobre las razones que justifican estos cambios tan abruptos de parte de los gobernantes, concluyendo que se trató de responder a las presiones de a lo menos una parte de los habitantes de Chaitén, que cansados por la incertidumbre, optaron por retornar a las mismas áreas que habían ocupado históricamente. Paradójicamente, en muchos casos volvieron a ocupar viviendas que les habían sido adquiridas por el gobierno como forma de asegurar que la ciudad sería abandonada totalmente y para siempre. Otra importante razón parece ser el altísimo costo de las inversiones públicas que habría que haber realizado, que superaría los 3.500 millones de dólares, algo difícil de aceptar por un gobierno de derecha que confía solo en la iniciativa privada como gestor del desarrollo.

Desconociendo la forma en que procederá la reinstalación y reconstrucción de la ciudad es imposible anticipar el corolario de estas erráticas actuaciones políticas y la manera específica en que han acrecentado o disminuido la vulnerabilidad y resiliencia social de los habitantes locales. Sin embargo, es posible observar que una parte de ellos a lo menos, se ha empoderado y accedido a recursos económicos, sociales, políticos y culturales, transformado el desastre en una oportunidad para acrecentar y diversificar sus bienes y lugares. Desde luego que ello no está exento de oportunismo y clientelismo político, lo que constituye una importante variable que se impone frente a cualquier consideración científica o técnica o respecto a los antecedentes sociodemográficos que se utilizan convencionalmente para representar las estructuras y procesos sociales en el análisis de los desastres socionaturales. 


\section{BIBLIOGRAFÍA}

Albornoz, C. y Romero, H. (2013): “Gestión pública en Chile frente a la emergencia volcánica. Erupciones de los volcanes Hudson (1991) y Chaitén (2008)”. IV Congreso Nacional de Geografía de Universidades Públicas y las XI Jornadas Cuyanas de Geografía, Mendoza - Argentina.

Albornoz, C., Salgado, M., Molina, G., Castro, F. y Rodríguez, A. (2013): “Conocimiento local y Percepción del riesgo volcánico de la población joven en la localidad de Chaitén (Chile)". Anales de la Sociedad Chilena de Ciencias Geográficas, 17-23.

Adger, N. (2003): "Social Capital, Collective Action, and Adaptation to Climate Change", Economic Geography 79 (4), 2003, 387-404.

Adger, N. (2006): "Vulnerability", Global Environmental Change 16, 268-281

Castree, N.; Demeritt, D. y Liverman, D. (2009): "Introduction: Making Sense of Environmental Geography", In A Companion to Environmental Geography, Edited by Noel Castree, David Demeritt, Diana Liverman and Bruce Rhoads. Wiley-Blackwell, 2009, 1-16.

Collins, T. (2009): "The production of unequal risk in hazardscapes: An explanatory frame applied to disasters at the US-Mexico border". Geoforum 40, 2009, 589-601.

Cutter, S., Boniff, B. y Lynn, S. (2003): "Social Vulnerability and Environmental Hazards. Social". Sciences Quartely, Vol.84, Number 2, June 2003, 242-261.

Cutter, S. y Emerich, C. (2006): "Moral Hazard, Social Catastrophe: The Changing Face of Vulnerability along the Hurricane Coast". Annals of the American Academy AAPPS, 604, 102-112.

Cutter, S.; Barnes, L.; Berry, M.; Burton, C.; Tate, E.; Webb, S. (2008): “A place-based model for understanding community resilience to natural disasters". Global Environmental Change 18, 2008, 598-606.

Dauphiné, A. y Provitolo, D. (2013): Risques et catastrophes: observer, spatialiser, comprendre, Paris, Armand Colin, $2^{\mathrm{e}}$ éd.

Demeritt, D. (2009): "From externality to inputs and interference: framing environmental research in Geography”.Transaction of British Geographers NS34, 2009, 3-11.

Eco-Universidad de Concepción (2011); Reconstruyéndonos. Nuestra Memoria, Nuestras Voces, Nuestro Futuro. Santiago de Chile, Imprenta Maval.

Hawkins, R. y Maurer, K. (2011): "You fix my community, you have fixed my life": the disruption and rebuilding of ontological security in New Orleans". Disasters 35(1) 2011, 143-59.

Hiroyuki, T. (2012): "Why do Victims of the Tsunami Return to the Coast?" International Journal of Japanese Sociology, March 2012 21, 21-29.

Macaya, P. (2012): "Volcán Chaitén. Consecuencias y Discusiones. Ensayo del curso de Teoría y Política Ambiental". Magister Governance of Risks and Resources. Universidades de Heildelberg, de Chile y Católica de Chile.

Marchant, J. (2010): Lágrimas de Ceniza. Estudio Cualitativo sobre el desplazamiento de los habitants de Chaitén asentados en las ciudades de la Isla de Chiloé y Puerto Montt. Proyecto de Práctica Profesional. Oficina Nacional de Emergencia (ONEMI), 
noviembre 2010, 165. Gobierno Regional de la Región de Los Lagos. Resolución Chaitén. Estado de Situación a noviembre de 2010.

McLaughin, P. y Aretz, S. (2008): "Structure, Agency and Environment. Towards and Integrated Perspective of Vulnerability". Global Env. Change 18, 2008, 99-111.

Mustafa, D. (2005): "The production of an urban hazardsacape in Pakistan: modernity, vulnerability, and the range of choice", Annals of the Association of American Geographers 95, 2005, 566-586.

Puc-Uach (Pontificia Universidad Católica de Chile-Universidad Austral de Chile) (2009): Estudio de Escenarios de Reconstrucción y Relocalización de la Ciudad de Chaitén, encargado por la Intendencia de la Región de Los Lagos. Vol. I, II y III, 2009.

Presidencia de la República de Chile (2009): Informe Encuesta Intención de Retorno. Comparación encuestas diciembre 2008-marzo 2009. Resumen Ejecutivo

Protege (Red de Protección Social del Gobierno de Chile) (2010): Proyecto Ciudad Chaitén. Ciudad Sustentable. Participación Comunitaria y Plan Maestro Ciudad de Chaitén. Informe Final

Romero, H., Moscoso, C. y Smith, P. (2009): "Lecciones y conclusiones sobre la falta de sustentabilidad ambiental del crecimiento espacial de las ciudades chilenas". En Chile: del país urbano al país metropolitano, Hidalgo, R., De Mattos, C., Arenas, F. (Editores). Serie GEOlibros $N^{\circ} 12$ Colección EURE-Libros. Instituto de Geografía e Instituto de Estudios Urbanos y Territoriales, Pontificia Universidad Católica de Chile, 89-110

Romero, H., Fuentes, C. y SMith, P. (2010): “Dimensiones geográficas territoriales, institucionales y sociales del terremoto de Chile de febrero 2010". Cuadernos de Geografía, Revista Colombiana de Geografía. Bogotá: Universidad Nacional de Colombia, $\mathrm{n}^{\mathrm{o}} 19,2010,135-150$.

Romero, H. y MendonçA, M. (2011): “Ondas de frío registradas en invierno de 2010: Necesidad de una perspectiva regional integrada para la Climatología latinoamericana”. Revista Geográfica de América Central, número especial EGAL, 2011-Costa Rica. II Semestre 2011, 1-13.

Romero, H.; MendonçA, M.; Méndez, M.; Smith, P. (2011a): "Multiescalaridade, Relações Espaciais e Desafios Ecológicos-sociais da Climatologia Sudamericana. O caso do Deserto de Atacama", Revista Brasileira de Climatologia. №. 8, 7-29. Disponible en: http://ojs.c3sl.ufpr.br/ojs/index.php/revistaabclima/article/view/25785

Romero, H.; Fuentes, C. y Smith, P. (2011b): "La Geografía de los Riesgos "Naturales" y el Terremoto de Chile del 27 de febrero de 2010". En Bocco, G., Urquilla., P. y Vieyra, A. (Coords.), Geografía y Ambiente en América Latina" Centro de Investigaciones en Geografía Ambiental, Universidad Nacional Autónoma de México. 2011b, 251-282.

Romero, H. y Mendonça, M. (2012): “Amenazas Naturales y Evaluación Subjetiva en la Construcción de la Vulnerabilidad Social ante Desastres Naturales en Chile y Brasil". Revista Internacional Interdisciplinar INTERthesis. Doutorado Interdisciplinar em Ciéncias Humanas. UFSC, SC, Brasil, Vol.09 Nº1, jan-jun 2012, 127-180 
Romero, H.; Méndez, M. y Smith, P. (2012): "Mining development and environmental injustices in The Atacama Desert (Northern Chile)", Environmental Justice, Vol.5, Number 2, Mary Ann Liebert Inc., 70-76.

Romero, H. y VidAL, C. (2014): “Expansión urbana y vulnerabilidad socio territorial ante desastres naturales de la conurbación Concepción-Talcahuano: inundaciones de julio de 2006 y Tsunami de febrero de 2010”. En L. Méndez (Editor) (2014): Geografía y Sociedad: El Gran Concepción su origen, su desarrollo urbano y su evolución social. Ediciones Universidad del Bío-Bío, Chile, 321-351.

Salgado, M. y Ugarte, A. M. (2012): “Concientización y Acción Política ciudadana en contextos de vulnerabilidad tras desastres socionaturales: el caso de Chaitén". Ponencia al Precongreso Latinoamericano de Sociología, Pucón, septiembre 2012.

Subdere, Subsecretaría de Desarrollo Regional y Administrativo del Gobierno de Chile, 2012. Estudio de riesgos de sismos, volcanismo, remoción en masa, inundación por desbordes de cauce y canales y maremotos para ocho localidades de la Comuna de Chaitén, Informe Final, Vol. I, II y III, febrero de 2012.

VidAl, C. y Romero, H. (2010): "Efectos ambientales de la urbanización de las cuencas de los ríos Biobío y Andalién sobre los riesgos de inundación y anegamiento de la ciudad de Concepción". Concepción metropolitano (AMC). Planes, procesos y proyectos. Pérez, L. e Hidalgo, R. (Editores), Serie GEOlibros No14, 2010, 287-304.

Zimmerer, K. (2003): "Environmental Zonation and Mountain Agriculture in Peru and Bolivia: Socioenvironmental Dynamic of Overlapping Patchworks and Agrobiodiversity Conservation", en K. Zimmerer, K. y Bassett, T. Political Ecology. An Integrative Approach to Geography and Environment, London, The Guilford Press, $137-158$ 
